\title{
Analysis of Arabidopsis thaliana HKT1 and Eutrema salsugineum/ botschantzevii HKT1;2 Promoters in Response to Salt Stress in Athkt1:1 Mutant
}

\author{
Ismat Nawaz ${ }^{1,2} \cdot$ Mazhar Iqbal $^{1,3} \mathbb{D} \cdot$ Henk W. J. Hakvoort ${ }^{4} \cdot$ Albertus H. de Boer $^{5} \cdot$ Henk Schat $^{1}$
}

Published online: 12 April 2019

(c) The Author(s) 2019

\begin{abstract}
Soil salinity imposes a serious threat to the productivity of agricultural crops. Among several other transporters, high-affinity $\mathrm{K}^{+}$transporter (HKT)'s play an important role in reducing the phytotoxicity of $\mathrm{Na}^{+}$. Expression of Eutrema salsugineum (a halophyte) $H K T 1 ; 2$ is induced upon salt exposure. To elucidate the role of its promoter, we compared the sequences of HKT1;2 promoters from E. salsugineum (1822 bp) and E. botschantzevii (1811 bp) with Arabidopsis thaliana HKT1;1 (846 bp) promoter. In silico analysis predicted several cis-acting regulatory elements (GT-1 elements, core motifs of DRE/ CRT, MYC/MYB-recognition sites and ACGT elements). Activities of the three promoters were analyzed by measuring $H K T 1 ; 1$ and/or HKTl;2 transcript level in the Athkt1;1 mutant plants. $\mathrm{NaCl}$ tolerance of the transgenics was also assessed. Our results depicted that expressing either AtHKTI;1 or EsHKT1;2 coding regions under the control of AtHKT1;1 promoter, almost reversed the hypersensitivity of the mutant for salt, on contrarily, when AtHKT1; 1 coding sequence expressed under either Es or EbHKT1;2 promoters did not. Changes in shoot $\mathrm{Na}^{+} / \mathrm{K}^{+}$concentrations under salt exposure is significantly consistent with the complementation ability of the mutant. The transcript concentration for genes under the control of either of Eutrema promoters, at control level was very less. This may suggest that either an important upstream response motif is missed or that A. thaliana misses a transcriptional regulator that is essential for salt-inducible HKTl expression in Eutrema.
\end{abstract}

Keywords $H K T 1 ; 2 \cdot$ Promoter swapping $\cdot$ RT-qPCR $\cdot$ In silico

\section{Introduction}

The detrimental effects of high salinity levels on plants are the consequence of osmotic and ionic stresses [1]. Plants with the ability to successfully complete the life cycle under

Electronic supplementary material The online version of this article (https://doi.org/10.1007/s12033-019-00175-5) contains supplementary material, which is available to authorized users.

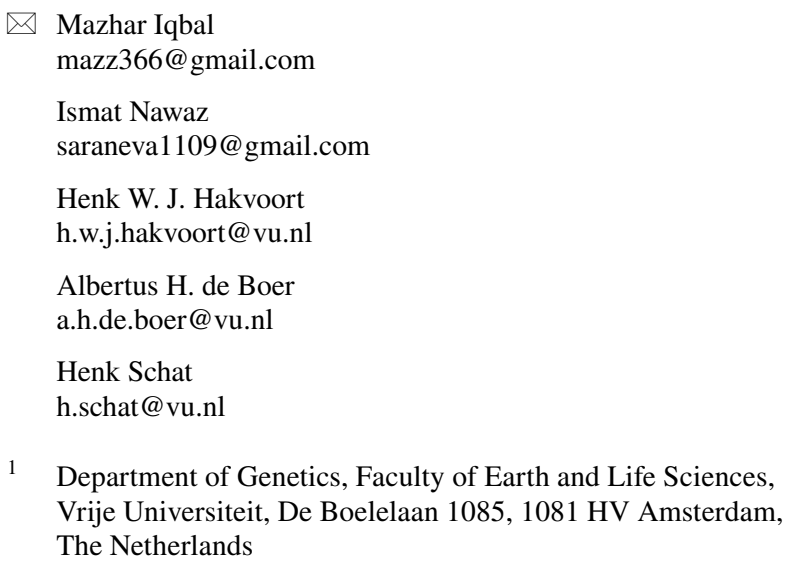

2 Department of Environmental Sciences, Biotechnology Program, COMSATS University Islamabad, Abbottabad Campus, Abbottabad, Pakistan

3 Department of Environmental Sciences, Faculty of Biological Sciences, Quaid-i-Azam University, Islamabad, Pakistan

4 Department of Systems Ecology, Faculty of Earth and Life Sciences, Vrije Universiteit, De Boelelaan 1085, 1081 HV Amsterdam, The Netherlands

5 Department of Structural Biology, Faculty of Earth and Life Sciences, Vrije Universiteit, De Boelelaan 1085, 1081 HV Amsterdam, The Netherlands 
continuous exposure to higher salinity stress $(>200 \mathrm{mM}$ $\mathrm{NaCl}$ ) are categorized as halophytes, however, rest plants are known as glycophytes (salt-sensitive) [2]. The mechanisms underlying high-level salt tolerance among many halophytes are not properly understood, but the trait is often, more or less implicitly, supposed to depend on enhanced capacities of cellular $\mathrm{Na}^{+}$and $\mathrm{K}^{+}$compartmentalization and homeostasis, or compatible organic osmolyte synthesis, through alterations of the expression patterns of genes encoding $\mathrm{Na}^{+} / \mathrm{K}^{+}$transporters or genes involved in the synthesis or breakdown of compatible solutes [2]. The $\mathrm{Na}^{+}$transporters, SOS1, NHX1 and HKT1 have often been considered to play key roles in salt tolerance in halophytes [3], however, comparisons between their expression patterns in halophytes and glycophytes are, with few exceptions [4-7], not available to date.

The HKT is $\mathrm{Na}^{+} / \mathrm{K}^{+}$transporter and gene family is increasingly studied for its role in $\mathrm{Na}^{+}$detoxification within the plant body [8-10]. Plett et al. [11] showed that overexpression of AtHKT1;1 in the mature root cortex improved the shoot $\mathrm{Na}^{+}$exclusion, and thus increased salinity tolerance in transgenics. In a recent study, Nawaz et al. [7] compared HKTl expression among halophytic and glycophytic species of Cochlearia, and found much higher expression levels in the halophytic species than in the glycophytic, supporting the hypothesis that enhanced $H K T l$ expression may be crucial for high-level salt tolerance, indeed. Eutrema salsugineum (formerly known as Thellungiella halophila), thought to be a suitable halophytic model plant because of its high DNA identity with $A$. thaliana [12], has at least three HKTl genes, HKTl;1, HKT1;2 and HKT1;3 [13]. EsHKT1;2, is a $\mathrm{K}^{+}$transporter, whereas EsHKT1;1 and EsHKT1;3 are $\mathrm{Na}^{+}$transporters, like AtHKT1;1 [13-15].

There are many reports suggesting the presence of an efficient transcriptional regulation of the salt-responsive genes in halophytic species. Zhang et al. [16] analyzed the expression pattern of Suaeda liaotungensis gene BADH (SlBADH) promoter. They used $300 \mathrm{bp}$ fragment (upstream from ATG) and showed almost six fold higher expression of gene under $400 \mathrm{mM} \mathrm{NaCl}$ stress as compared to control. Sun et al. [17] compared the activities of two promoters from A. thaliana and $T$. halophila gene (Vacuolar $H^{+}$-Pyrophosphatase-VP) and concluded that $130 \mathrm{bp}$ of the ThVPl promoter contains cis-acting regulatory elements (two enhancers) which are involved in strong induction of the gene under salt stress. Li et al. [18] analyzed the role of PEAMT gene promoter from Suaeda liaotungensis (a halophyte) and predicted the presence of many salt-responsive cis-element ( $897 \mathrm{bp}$ upstream from start codon). They reported the enhanced GUS activity (18-fold) under the control of SIPEAMT promoter at $200 \mathrm{mM}$ of $\mathrm{NaCl}$ stress, in the transgenic tobacco. These studies and many others provided a notion that even a small fragment of promoter can contain salt stress-inducible, cisacting motifs to regulate the expression of different genes, upon salt stress.

Surprisingly, very little work has been done to explore the role of the $H K T 1$ promoter in the transcriptional regulation of the gene. Asins et al. [19] has observed the altered expression of SlHKTI;1 gene in roots and leaves of NILs due to the difference in their promoter sequences, which affects the transport activity of the gene, as well. The cisregulatory allelic variations among various Arabidopsis ecotypes has also been documented for the AtHKTl; 1, which lead to differential salinity tolerance of the ecotypes [20, 21]. However, there is not even a single study to compare the promoter activities of a halophyte (like Eutrema salsugineum/botschantzevii) and a glycophyte (like A. thaliana). In this study, we performed an in silico analysis of three HKT1 promoter sequences, i.e., A. thaliana (Pro $_{A t H K T 1 ; 1}$, 1822 bp), Eutrema salsugineum (=halophila), ecotype Shandong $\left(\right.$ Pro $\left._{\text {EsHKTI;2, }}, 1822 \mathrm{bp}\right)$, and Eutrema botschantzevii (formerly known as Thellungiella botschantzevii), ecotype Saratov (Pro $\left.{ }_{E b H K T 1 ; 2}, 1822 \mathrm{bp}\right)$. We compared the activities of four promoter sequences, i.e., Pro $_{A t H K T 1 ; 1}(846 \mathrm{bp}$ ), Pro $_{E s H K T 1 ; 2}(1822 \mathrm{bp})$, Pro $_{E b H K T l ; 2}(1811 \mathrm{bp})$, and the CaMV 35S promoter, through examining AtHKTI;1 and EsHKT1;2 gene expression in the A. thaliana hktl;1 mutant background. We also compared the potential of the constructs to reverse the $\mathrm{Na}^{+}$hypersensitivity phenotype of the Athkt $1 ; 1$ mutant.

\section{Materials and Methods}

\section{Plant Materials and Experimental Conditions}

Eutrema salsugineum and E. botschantzevii seeds, originating from a coastal area near Shandong, China, and a solonchak soil in Saratov, Russia, were sown on garden soil (Jongkind B.V., Number 6, Aalsmeer, The Netherlands). When seedlings were three-weeks old, they were transfered to hydroponics culture [22]. Mature plants were harvested which were snap-frozen. For subsequent DNA/RNA extraction, samples were stored at $-80^{\circ} \mathrm{C}$ [23].

Surface sterilization of different types of seeds [Arabidopsis thaliana (Col) wild type, Athkt1;1 mutants and transgenic lines] were done using $96 \%$ ethanol and $10 \%$ bleach, followed by three times washing in distilled water. Surface-sterilized seeds were mixed in agarose $(0.1 \%)$ and spread on square petri plate containing gelrite $(0.8 \% \mathrm{w} / \mathrm{v})$ with Murashige and Skoog (MS-0.5\%) salt, whose $\mathrm{pH}$ was adjusted around 5.7-5.9. For transgenic lines, hygromycin ( $25 \mu \mathrm{g} / \mathrm{ml})$ and for the Athkt $1 ; 1$ mutants, $25 \mu \mathrm{g} / \mathrm{ml}$ kanamycin was used as selective marker. Photoperiod for seeds germination was $10 \mathrm{~h}$ light and $14 \mathrm{~h}$ dark, at $22^{\circ} \mathrm{C}$ temperature. 
When seedlings developed their true leaves, they were transplanted to hydroponics 1-1 pots, having half-strength modified Hoagland's nutrient solution [22] in climate room. Light intensity in climate room was set $220 \mu \mathrm{mol} / \mathrm{m} / \mathrm{s}$ at the plant level, along with $20{ }^{\circ} \mathrm{C}$ (day), $15^{\circ} \mathrm{C}$ (night) temperature and $75 \%$ relative humidity. Hydroponics solutions were renewed two times a week. When plants get adapted to the new environment (almost after two weeks), ten plants from each type were exposed to $\mathrm{NaCl}(50 \mathrm{mM})$ using control as a reference. Salt stress was increased gradually (at first $25 \mathrm{mM}$ stress was given then increased to $50 \mathrm{mM}$ ). Plants were harvested after two weeks of the final concentration. Fresh weight of the roots and shoots in gram was taken.

\section{RNA/DNA Extraction and First-Strand cDNA Synthesis}

Frozen shoot tissues were used for RNA extraction via Trizol $^{\mathrm{TM}}$ (Invitrogen) method, as described in Jack et al. [23]. For the synthesis of single-stranded cDNA, total RNA $(2.5 \mu \mathrm{g})$ which was boiled for $1 \mathrm{~min}$, was used. Reverse transcription was accomplished with M-MLV Reverse Transcriptase (100 Units), 100 mM DTT, 2 mM dNTPs, RT buffer $(10 \times)$ and $10 \mu \mathrm{M}$ of oligo dT primer (Invitrogen). For DNA isolation, we used the protocol as described in Rivera et al. [24].

\section{Es/EbHKT1;2 Promoters Sequencing, Constructs Making and the Transformation of the Athkt 1;1 Mutant}

The sequence of $H K T 1 ; 2$ promoters from E. salsugineum and E. botschantzevii were obtained by chromosome walk, using Universal Genome Walker ${ }^{\mathrm{TM}}$ kit by Clontech. Gene specific reverse primers were used for chromosome walk on DNA (genomic). 1822 bp from E. salsugineum, 1811 bp from $E$. botschantzevii and $846 \mathrm{bp}$ from $A$. thaliana [25] upstream from start codon (ATG) of $H K T 1$, were used as promoters. Following constructs were prepared: $\operatorname{Pro}_{355}:$ EsHKT1;2, Pro $_{\text {AtHKT } 1 ; 1}:$ EsHKT $1 ; 2, \quad$ Pro $_{\text {AtHKT } 1 ; 1}:$ AtHKT $1 ; 1$, Pro $_{\text {EsHKT } ; 2}: \because$ AtHKT1; 1 and Pro $_{\text {EbHKTl;2 }} \because$ AtHKT1;1. PCR's were performed using "Phusion ${ }^{\circledR}$ DNA Polymerase" by Finnzymes, on gDNA for amplification of the promoter region and on cDNA for amplification of $H K T$ coding sequences. Forward primers of Pro $_{A t H K T 1 ; 1}$, Pro $_{\text {EbHKT1;2}}$, Pro $_{\text {EsHKT1;2 }}$ and Pro $_{\text {EsHKT1;2 }}: \because$ AtHKT1; 1 contained "CACC" 5 ' overhang which is necessary for directional cloning in pENTR/D Topo, while primers for Pro $_{A t H K T 1 ; 1}:$ AtHKT1; 1, Pro $_{A t H K T 1 ; 1}::$ EsHKT1;2 and Pro $_{\text {EbHKT1;2: }: A t H K T 1 ; 1}$ contained attB1/2 sites (Table S1: Supplementary Information). BP reaction was performed using BP Clonase $®$ II enzyme mix between vector containing attP-site and DNA fragment having attB-flanked to generate an entry clone. This entry clone was subsequently utilized for

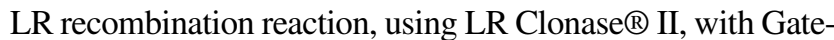
way ${ }^{\circledR}$ destination vector for the development of an expression vector. Following expression vectors; pH7WG2, pH7WG2 $\left(-\mathrm{PrO}_{35 \mathrm{~S}}\right)\{$ constructed from $\mathrm{pH} 7 \mathrm{WG} 2\}$ and pHGWFS7 [26], were used. pHGWFS7 was used for the promoters analysis, however, pH7WG2 (-Pro $\left.{ }_{35 \mathrm{~S}}\right)$ was used for the constructs (having a promoter). Agrobacterium tumefaciens strain C58 (pMP90) containing specific construct was used to transform A. thaliana hkt1;1 mutant.

\section{Screening of Transgenic Lines}

Athkt1;1 mutants (Col) seeds, obtained from NASC stock center (N6531), and A. thalaina wild type seeds were sown on the garden soil (Jongkind B.V., Number 6, Aalsmeer, The Netherlands). Plants of Athkt $1 ; 1$ mutants, at silique forming stage, were transformed using 'flower dipping' protocol as described by Clough and Bent [27]. The matured seeds were harvested from $T_{0}$ plants and were sowed on square Petri plates containing MS media, after surface sterilization. The transformed plants were easily distinguished from non-transformed ones, after 2 weeks depending on root growth. Transformed plants were transplanted to hydroponics culture [22]. After 2 weeks, leaves and root samples were taken for RNA extraction, from selected $T_{1}$ progeny. After selection, null segregants were never found, and selected plants were supposed to be a mixture of heterozygotes and homozygotes. Transcript levels of the genes (Actin-2 as reference gene) were measured by Real-Time quantitative PCR. Primers for RT-qPCR were designed with a $\mathrm{G} / \mathrm{C}$ ratio ranging between 50 and $60 \%$ with $T_{\mathrm{m}}$ (melting temperature) range between 58 and $60{ }^{\circ} \mathrm{C}$. Genespecific primers for Eutrema salsugineum (EsHKT1;2), Eutrema botschantzevii (EbHKT1;2), A. thaliana (AtHKT1;1) and Act-2 (as an internal control for expression analysis) were designed separately (Table S2: Supplementary Information). RT-qPCR data were analyzed as described by Livak and Schmittgen [28]. The gene expression was normalized to the highest expression, which was assigned a value of 1 .

\section{Tolerance Index and Water Content}

At the time of harvest, fresh weight of root and shoot samples were taken. Samples were air dried at $65^{\circ} \mathrm{C}$ for $72 \mathrm{~h}$. Tolerance index was calculated over ten biological replicates of each transgenic line using the formula:

Tolerance Index $(\mathrm{TI})=\frac{\text { Average fresh weight at } 50 \mathrm{mM}}{\text { Average fresh weight at the control }}$

The percentage of water was calculated using the following formula:

$\%$ age of water $=\left(\frac{\text { Fresh weight }- \text { Dry weight }}{\text { Fresh weight }}\right) \times 100$ 


\section{Measurement of $\mathrm{Na}^{+}$and $\mathrm{K}^{+}$}

After taking fresh weight, the roots of two plants were pooled together while shoots were dried and stored separately. After measuring shoot dry weight two shoots were pooled together like roots. $\mathrm{Na}^{+}$and $\mathrm{K}^{+}$concentrations, in roots and shoots were determined (two plants pooled, to make five samples per genotype, per treatment level) by extracting $20 \mathrm{mg}$ of dry material in $2 \mathrm{ml}$ eppendorfs. Two milli liters of water was added in each eppendorf then boiled it for $1 \mathrm{~h}$ at $90{ }^{\circ} \mathrm{C}$ in a water bath. After cooling, samples were filtered and dilutions were made, if needed. After proper dilution, $\mathrm{Na}^{+}$and $\mathrm{K}^{+}$analysis on Atomic Absorption Spectrophotometer (AAS100) was done.

\section{Results}

\section{In Silico Analysis of EsHKT1;2, EbHKT1;2 Promoters in Comparison with the AtHKT1;1 Promoter}

The sequences of the EsHKT1;2, EbHKT1;2 and AtHKT1;1 promoters (each was $1822 \mathrm{bp}$ in length) were used for in silico analysis via web-based tools (PLACE/Signal Scan database-http://www.dna.affrc.go.jp/) [29]. The promoters from EsHKT1;2 and EbHKT1;2 have 95\% identity among themselves, while both promoters showed 38\% identity with the AtHKT1; 1 promoter, on nucleotide basis (sequence alignment S1: Supplementary Information).

We identified several putative cis-acting regulatory biotic/abiotic stress-responsive elements which include the core motif for DRE/CRT (dehydration-responsive element/C-repeat) and putative GT-1 cis-elements (2 motifs in EsHKT1;2 and EbHKTl;2 and 3 in the AtHKT1;1 promoter sequences; Table S3: Supplementary Information). A common cis-acting element, CAAT-box, which is mostly present in promoters and enhancer regions, was identified at several places in three promoter sequences. The ACGT motif was found 3, 4 and 5 times in AtHKT1;1, EbHKT1;2 and $E s H K T 1 ; 2$ promoter sequences, respectively. Potential MYB-, MYC- and MYBCORE recognition sites have been identified at several places in the analyzed sequences (Table S3: Supplementary Information).

\section{Selection and Molecular Analysis of $T_{0} / T_{1}$ Transgenic Plants}

The amplified E. salsugineum cDNA sequence appeared to be EsHKT1;2. It shared $83 \%$ identity, on a nucleotide basis, with $A$. thaliana HKT1; 1 (sequence alignment $\mathrm{S} 2$ : Supplementary Information). On a protein basis, EsHKT1;2 and AtHKT1; 1 were 79\% identical (sequence alignment S3: Supplementary Information). The EbHKT1;2 (1811 bp) and
EsHKT1;2 (1822 bp) promoters were cloned for this experiment. We successively performed two experiments with two sets of independent transgenic lines.

PCR and Real-Time PCR analyses were performed on all the $T_{0}$ plants and 3-4 randomly selected plants from the $T_{1}$ progeny (the first generation of transgenic plants) to determine the expression levels (Fig. 1) of the transgenes, under control condition. Only those transgenic lines were selected which showed the similar transcript expression level of AtHKT1;1 and/or EsHKT1;2, for the subsequent experiments (at least two independent lines per genotype). EsHKT1;2 was most strongly expressed from Pro $_{A t H K T 1 ; 1}$, in comparison with any other promoter (Fig. 1). Also AtHKT1; 1 was well expressed, approximately at the level of wild type $A$. thaliana, when expressed under its native promoter. The

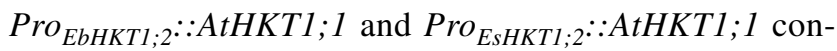
structs were not detectably expressed, under un-stressed condition.

\section{Tolerance Index}

There were evident differences in salt tolerance among various transgenic lines. A. thaliana wild type and transgenic under the control of $\mathrm{Pro}_{A t H K T I ; 1}$ and $\mathrm{Pro}_{35 S}$ looked green and remained healthy. There were no apparent foliar chlorosis/ necrosis, however, they showed senescence of the cotyledons and the oldest leaves ( 10 to $20 \%)$. These transgenic lines did not exhibit any mortality upon exposure to $50 \mathrm{mM}$ $\mathrm{NaCl}$. Transgenic lines under the control of Pro $_{\text {EsHKTI;2 }}$ or Pro $_{E b H K T 1 ; 2}$ proved to be sensitive with much smaller leaves. These lines exhibited chlorosis/necrosis with enhanced senescence of the older leaves ( 40\%), as well.

Under exposure to $50 \mathrm{mM} \mathrm{NaCl}$ both of the constructs with the A. thaliana promoter, $\operatorname{Pro}_{A t H K T 1 ; 1} \because A t H K T 1 ; 1$ and Pro $_{A t H K T 1 ; 1}:$ EsHKT1;2, complemented the Athkt1;1 mutant, approximately to wild type level (Fig. 2). As expected, the constructs with extremely low expression, did not complement the Athkt1;1 mutant to any significant extent.

The Pro $_{355}:$ :EsHKT1;2 constructs complemented the mutant in one of the experiments, but not in the other, in spite of the fact that in both experiments the $H K T$ expression level was at least one order of magnitude higher under the CaMV 35S promoter than under any of the others. Overall, the results of the two experiments were consistent (Fig. 2).

\section{The Water Content of Fresh Leaves}

Consistent with their tolerance index, lines harbouring the Pro $_{A t H K T 1 ; 1}: \because E s H K T 1 ; 2$ and Pro $_{A t H K T 1 ; 1} \because$ AtHKT1;1 constructs maintained wild type-like water percentages in their leaves (>90\%) under salinity stress, whereas the plants with the Eutrema promoters desiccated to a degree comparable with that of the Athkt1;1 mutant (Fig. 3). The 

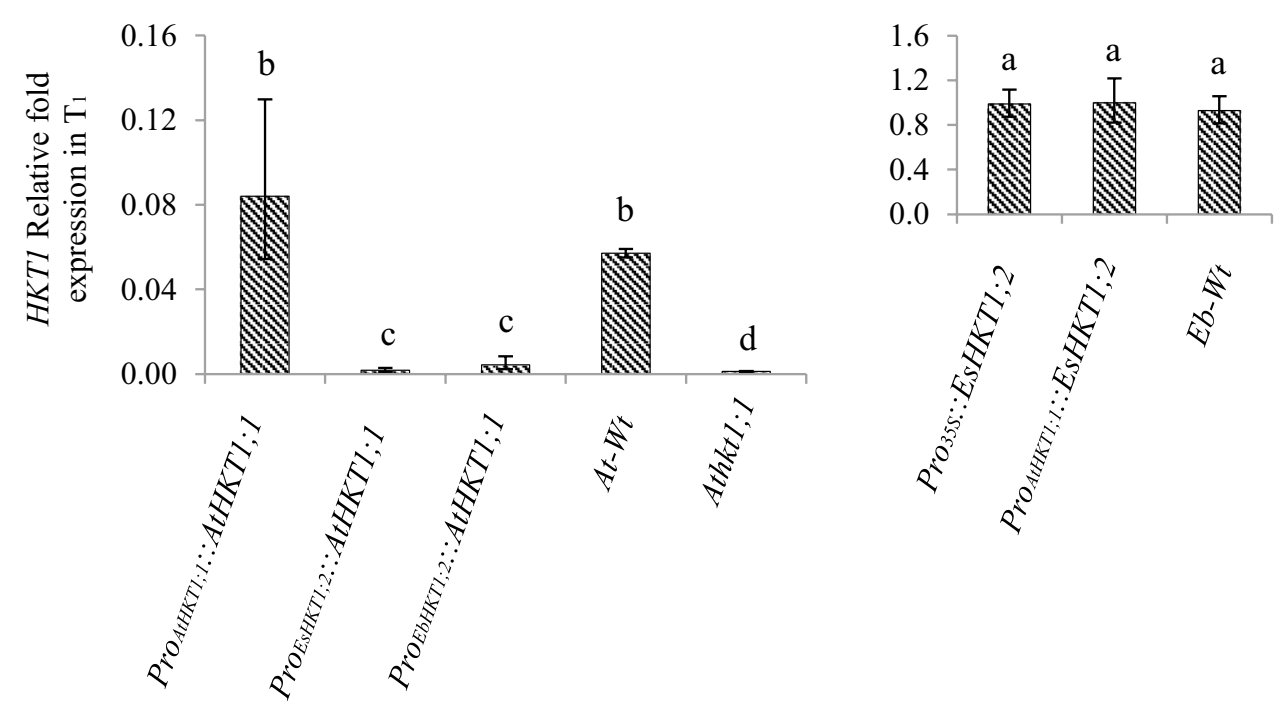

Fig. 1 Expression of $H K T 1$ in transgenic lines $\left(T_{1}\right)$ harbouring

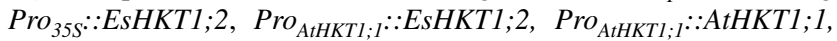

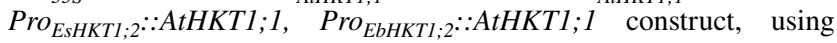
Real-Time quantitative PCR. A. thaliana wild type (At-Wt) and Athkt1;1 mutant were used as positive and negative controls, respec-

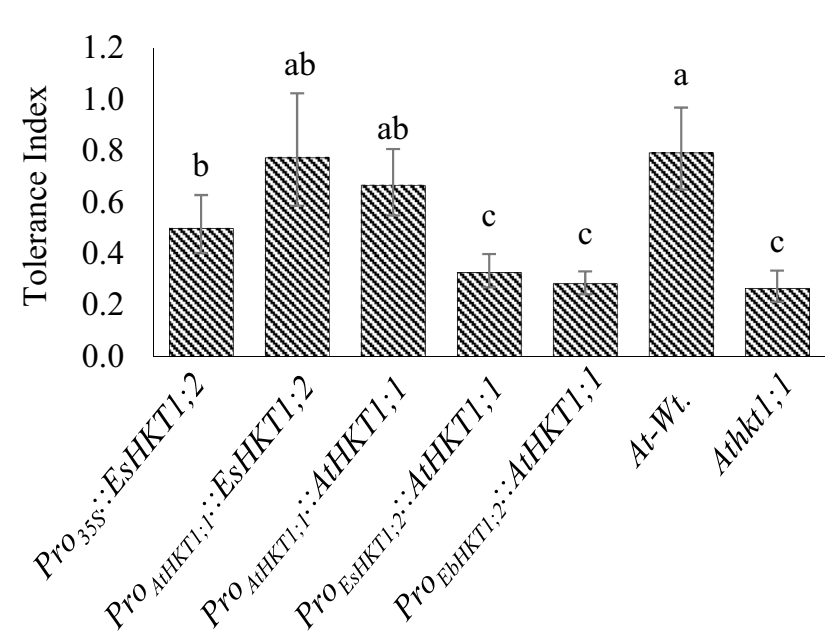

Fig. 2 Tolerance index on fresh weight basis measured in $T_{1}$ progeny harbouring $\quad \operatorname{Pro}_{35 S}:$ EsHKT1;2, $\operatorname{Pro}_{\text {AtHKTl; } 1:}:$ EsHKT1;2,

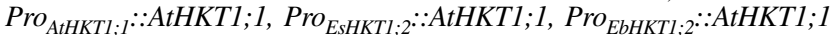
constructs. Plants were grown hydroponically at $50 \mathrm{mM} \mathrm{NaCl}$ stress for 14 days. A. thaliana wild type (At-Wt) and Athkt1;1 mutant were used as positive and negative controls, respectively. Ten replicates were used for each genotype and two independent $T_{1}$ lines were used in the experiment. Each bar is a mean of 20 plants \pm SE. Significant differences $(p<0.05)$ between means are indicated by different superscripted letters

construct with the CaMV 35S-promoter completely complemented the mutant in the first experiment, but only incompletely in the second one. Overall, the results of the two experiments were consistent. tively. Transgenic lines with comparable levels of $H K T 1$ expression were used in subsequent experiments. Each value is an average of 3-4 independent biological replicate \pm SE. Significant differences $(p<0.05)$ between means are indicated by different superscripted letters

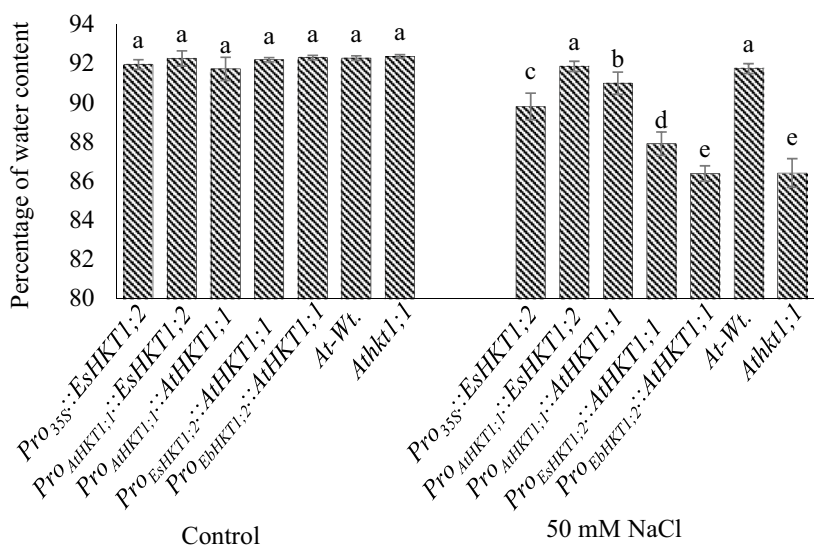

Fig. 3 Water content (percentage of shoot fresh weight) in $T_{1}$ progeny harbouring $\operatorname{Pro}_{355}:$ EsHKT1;2, $\operatorname{Pro}_{A t H K T 1 ; 1}:$ EsHKT1;2, Pro $_{\text {AtHKTl } ; 1} \because$ AtHKT1; 1, Pro $_{\text {EsHKTl } ; 2}: \because$ AtHKT1; 1, Pro $_{\text {EbHKTl } ; 2} \because$ AtHKT1; 1 constructs. Plants were grown hydroponically at control and $50 \mathrm{mM}$ $\mathrm{NaCl}$ stress for 14 days. A. thaliana wild type (At-Wt) and Athkt1;1 mutant were used as positive and negative controls, respectively. Ten replicates were used for each genotype and two independent $T_{1}$ lines were used in the experiment. Each bar is a mean of 20 plants \pm SE. Significant differences $(p<0.05)$ between means are indicated by different superscripted letters

\section{$\mathrm{Na}^{+}$and $\mathrm{K}^{+}$Analysis in Transgenic $T_{1}$ Plants}

We compared $\mathrm{Na}^{+}$and $\mathrm{K}^{+}$accumulation in shoots and roots of wild type and transgenic lines, in two sets of experiment. Average values of $\mathrm{Na}^{+}$contents from both experiment were used to depict the results (Fig. 4). In both experiments the 


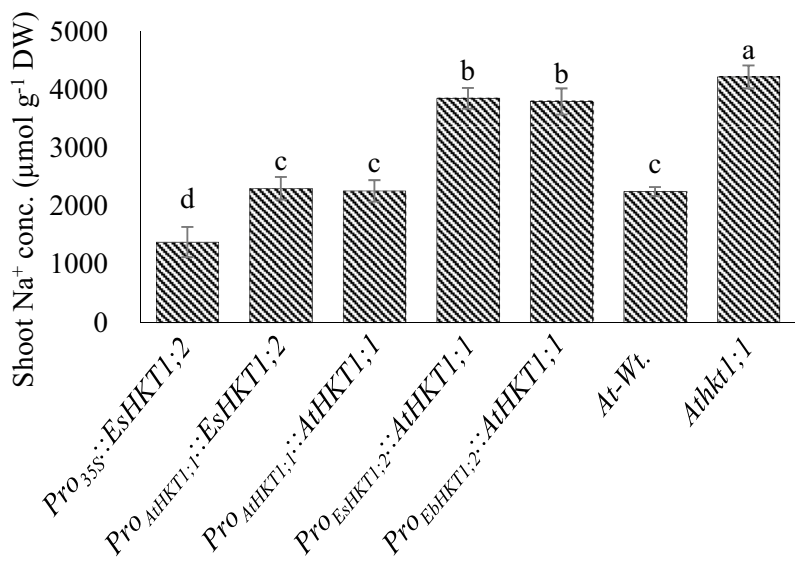

Fig. $4 \mathrm{Na}^{+}$concentrations in shoots of $T_{1}$ progeny from

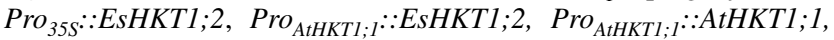

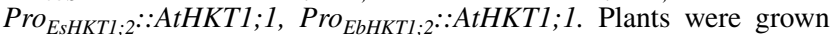
hydroponically at control and $50 \mathrm{mM} \mathrm{NaCl}$ stress for 14 days. A. thaliana wild type (At-Wt) and Athkt1;1 mutant were used as positive and negative controls, respectively. Five replicates (two shoots were pooled together) were used for each genotype and two independent $T_{1}$ lines were used in the experiment. Each bar is a mean of 10 plants \pm SE. Significant differences $(p<0.05)$ between means are indicated by different superscripted letters

genotype with the Pro $_{355}:$ EsHKT1;2 constructs exhibited a lower $\mathrm{Na}^{+}$concentration in their shoots than any of the other lines. The foliar $\mathrm{Na}^{+}$concentration in the Pro $_{A t H K T 1 ; 1} \because$ AtHKT1;1 and Pro $_{A t H K T 1 ; 1}: \because$ EsHKT1;2 lines was significantly higher than in the $\operatorname{Pro}_{35 s}:$ EsHKT1;2 line, but significantly lower than in the lines under either $E s$ and/ or $E b$ promoters. Although in second experiment, the $\mathrm{Na}^{+}$ concentration was detected comparatively higher in all genotypes, however, the trend of $\mathrm{Na}^{+}$contents remained the same as in both experiments. Overall, results from both experiments exhibited the similar trend (Fig. 4).

The Athkt1;1 mutant and transgenic lines under either Es and/or $E b$ promoters showed similar shoot $\mathrm{K}^{+}$concentration, however, wild type showed highest foliar $\mathrm{K}^{+}$concentration than all the other lines, under $\mathrm{NaCl}$ exposure (Fig. 5). Transgenic line expressing construct $\operatorname{Pro}_{A t H K T 1 ; 1} \because$ EsHKT1;2 also maintained significantly higher $\mathrm{K}^{+}$, upon salt stress, in its shoot as compared to the lines expressing $\operatorname{Pro}_{A t H K T 1 ; 1}: \because A t$ HKTI; 1 .

$\mathrm{Na}^{+} / \mathrm{K}^{+}$ratios of shoots and roots of all genotypes were also calculated (Supplementary Figure S1). Transgenic lines under the control of $E s$ and/or $E b$ promoter showed $\mathrm{Na}^{+} / \mathrm{K}^{+}$ratio in shoot as high as found for Athkt 1;1 mutants and was significantly higher as compared to all other genotypes (Supplementary Figure $\mathrm{S} 1 \mathrm{a}$ ). Root $\mathrm{Na}^{+} / \mathrm{K}^{+}$ratio for lines harbouring constructs Pro $_{A t H K T 1 ; 1}:$ EsHKT1;2, Pro $_{\text {EsHKTl } ; 2}:$ AtHKT1; 1 , Pro EbHKTl;2: $_{\text {AtHKT } 1 ; 1 \text { and }}$ Athkt1;1 mutant, were found significantly low as compared to other genotypes (Supplementary Figure S1b).

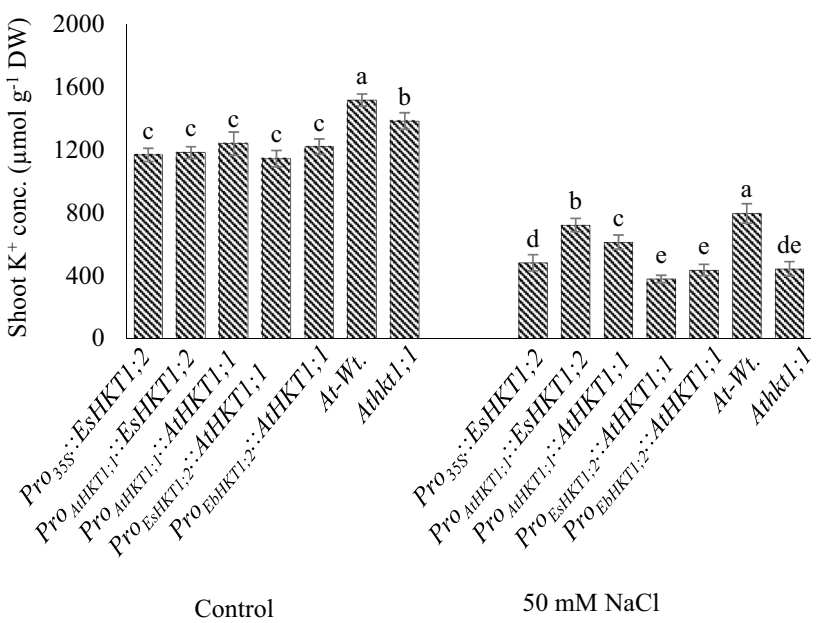

Fig. $5 \mathrm{~K}^{+}$concentration in the shoots of $T_{1}$ progeny from

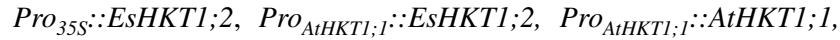
Pro $_{\text {EsHKTI } ; 2}: \because A t H K T 1 ; 1$, Pro $_{\text {EbHKTI } ; 2}: \because$ AtHKT1; 1 . Plants were grown hydroponically at control and $50 \mathrm{mM} \mathrm{NaCl}$ stress for 14 days. $A$. thaliana wild type (At-Wt) and Athkt1;1 mutant were used as positive and negative controls, respectively. Five replicates (two shoots were pooled together) were used for each genotype and two independent $T_{1}$ lines were used in the experiment. Each bar is a mean of 20 plants \pm SE. Significant differences $(p<0.05)$ between means are indicated by different superscripted letters

\section{Discussion}

In this study, we compared the expression pattern and activities of four promoters, i.e., Pro $_{A t H K T 1 ; 1}$, Pro ${ }_{E s H K T 1 ; 2}$, Pro $_{\text {EbHKTI:2 }}$ and CaMV 35S promoter, using AtHKT1;1 and EsHKT1;2 cDNA. As clearly shown by the tolerance index, only the $\operatorname{Pro}_{A t H K T 1 ; 1}: \because A t H K T 1 ; 1$ and Pro $_{A t H K T 1 ; 1}: \because E s H K T 1 ; 2$ constructs more or less completely complemented the Athkt1;1 mutant regarding its salt hypersensitivity phenotype. The $\operatorname{Pro}_{E s H K T 1: 2}: \because A t H K T 1 ; 1$ and Pro $_{\text {EbHKTI;2 }}:$ AtHKT1; 1 constructs did not yield any detectable complementation at all, while the $\operatorname{PrO}_{355}:$ EsHKT1;2 construct only incompletely complemented the mutant. The same conclusion can be drawn on the basis of the foliar water contents in the salt treatment. The complete lack of complementation observed with the Pro EsHKTI,2 $_{2} \because$ AtHKT1; 1 and Pro $_{E b H K T 1 ; 2}: \because$ AtHKT1;1 constructs is doubtlessly owing to low AtHKT1; 1 expression in the transgenic lines. The latter could be due to the absence of essential transcriptional activator/s or, more likely, the lacking an essential response element located upstream of the nucleotides that we used as promoter [30], which are required in A. thaliana. The incomplete complementation provided by the Pro $_{355}:$ EsHKT1;2 construct is most probably owing to a non-tissue-specific expression of EsHKT1;2 [8].

Our observation that AtHKTI;1 and EsHKT1;2 are both able to complement the Athkt1;1 mutant is not self-evident, since AtHKT1;1 is a $\mathrm{Na}^{+}$-selective transporter [31], whereas 
EsHKT1;2 is a $\mathrm{K}^{+}$-selective transporter, even in the presence of $\mathrm{NaCl}$ [14]. AtHKT1;1 is supposed to provide salinity tolerance through resorbing $\mathrm{Na}^{+}$from the xylem, thus preventing its accumulation in the shoot $[32,33]$, while EsHKT1;2 is supposed to maintain a sufficient $\mathrm{K}^{+}$concentration in shoot, under salinity stress (Fig. 5) [14, 15]. Foliar $\mathrm{Na}^{+}$concentrations were significantly lower for the wild type $A$. thaliana and lines having gene under AtHKTI; 1 promoter, surprisingly regardless of the origin of the coding region, thus both genes doubtlessly complemented the Athkt1;1 mutant under our experimental conditions. Which suggested that maintenance of sufficient $\mathrm{K}^{+}$concentration in shoot might be crucial for the salinity response of the transgenic line expressing Pro $_{A t H K T 1 ; 1}: \because$ EsHKT1;2 [34, 35]. However, the lines with constructs under $E s$ and/or $E b$ promoters showed the foliar $\mathrm{Na}^{+}$contents as high as in the Athkt1;1 mutant plants and failed to revert the $\mathrm{Na}^{+}$hypersensitivity of the mutants. Lines having construct under AtHKT1;1 promoter showed comparatively high $\mathrm{K}^{+}$concentrations as compared to the lines with the construct under $E s$ and/or $E b$ promoters, however, the difference was not too high between all lines.

Low-cytosolic $\mathrm{Na}^{+} / \mathrm{K}^{+}$ratio is a major determinant for the salt stress tolerance of any plant which ultimately play important role in growth and developmental success of the plant. Lower $\mathrm{Na}^{+} / \mathrm{K}^{+}$ratio in shoots of the lines harbouring construct Pro $_{355}::$ EsHKT1;2, Pro ${ }_{A t H K T 1 ; 1}::$ EsHKT1;2 and $\operatorname{Pro}_{A t H K T 1 ; 1}: \because A t H K T 1 ; 1$ as well as At-Wt. Positively correlate with salinity tolerance of these genotypes. Low $\mathrm{Na}^{+} / \mathrm{K}^{+}$ratio in shoot depicting plants ability to restrict more $\mathrm{Na}^{+}$contents in roots, and has been proved as a determinantal factor in Zea mays L. salt tolerance [36]. Low $\mathrm{Na}^{+} / \mathrm{K}^{+}$ratio in roots of transgenic lines with Pro $_{A t H K T 1 ; 1}::$ EsHKT1;2 indicate the higher contents of $\mathrm{K}^{+}$. Surprisingly, Pro $355:$ EsHKT1;2 showed high $\mathrm{Na}^{+} / \mathrm{K}^{+}$ratio, which may be attributed to ectopic expression of EsHKT1;2 under CaMV 35S promoter [8]. Overall, $\mathrm{Na}^{+} / \mathrm{K}^{+}$ratio in roots of other genotypes did not correlate positively with their salinity tolerance.

We identified many putative $c i s$-acting regulatory abiotic stress-responsive elements. The identified core motif related to dehydration-responsive element/C-repeat (DRE/CRT), has been reported for its role in drought and high salt-stress conditions [37]. Another important cis-acting regulatory element, GT-1 was also found which has been predicted to be involved in gene induction (SCaM-4) under salt stress, in Glycine max [38]. Identified CAAT-box and ACGT cisacting DNA sequences have been reported to be required for induction of various genes such as dehydration-responsive gene (AtNCED3) [39, 40]. Prabu and Parsad [41] demonstrated the putative role of Saccharum officinarum MYB gene and showed that the promoter activity was increased by 2 - to 4 -fold under salt, cold and dehydration stress. The indentified putative cis-acting elements (ACGT and MYC) were present in higher number in the promoter sequence of
EbHKT1;2, whereas the GT-1 cis-element, CAAT-box and MYBCORE were present at higher numbers in A. thaliana. Baek et al. [30] have reported two tandem repeats (R1 and $\mathrm{R} 2)$ in $A t H K T 1 ; 1$ promoter region ( $3.9 \mathrm{~kb}$ upstream of the start codon) and suggest that these repeats are involved in AtHKT1;1 regulation under salt stress. Unfortunately, we could not include longer fragment of AtHKTl;1 promoter however, expression of AtHKT1;1 transcripts in transgenic lines harboring AtHKT1;1 under the control of its native promoter (0.846 kb from ATG), was comparable to wild type expression level (Fig. 1). This indicates that $0.846 \mathrm{~kb}$ of AtHKT1; 1 promoter is functionally active and is complementing the Athkt1;1 mutant up to wild type level (Fig. 2). In any case, the role of above mentioned identified cis-acting elements in the Eutrema $H K T 1 ; 2$ promoters, if any, should be established by a series of promoter-deletion experiments.

\section{Conclusions}

Expression under the E. salsugineum and E. botschantzevii promoters did not yield any significant expression, either because of the lack of an essential upstream response element/s, or lacking an essential trans-acting regulator/s, in A. thaliana, and thus failed to complement the Athkt1;1 mutant. When the gene is expressed under AtHKT1; 1 promoter, both AtHKTI;1 and EsHKT1;2 fully complemented the mutant, in that they restored a wild type-like salt tolerance level. Several abiotic stress-responsive element have been identified in EsHKT1;2 and EbHKT1;2 promoter regions.

Acknowledgements We thank the Higher Education Commission (HEC), Pakistan, for the financial support of the first and second author under the programme "Overseas scholarship for MS/MPhil leading to $\mathrm{PhD}$ in selected fields Phase-II, Batch-I (90\%)".

Open Access This article is distributed under the terms of the Creative Commons Attribution 4.0 International License (http://creativeco mmons.org/licenses/by/4.0/), which permits unrestricted use, distribution, and reproduction in any medium, provided you give appropriate credit to the original author(s) and the source, provide a link to the Creative Commons license, and indicate if changes were made.

\section{References}

1. Munns, R., \& Tester, M. (2008). Mechanisms of salinity tolerance. Annal Review of Plant Biology, 59, 651-681. https://doi. org/10.1146/annurev.arplant.59.032607.092911.

2. Flowers, T. J., \& Colmer, T. D. (2008). Salinity tolerance in halophytes. New Phytologist, 179(4), 945-963. https://doi.org/10.11 11/j.1469-8137.2008.02531.x.

3. Ashraf, M., \& Akram, N. A. (2009). Improving salinity tolerance of plants through conventional breeding and genetic engineering: 
An analytical comparison. Biotechnology Advances, 27(6), 744752. https://doi.org/10.1016/j.biotechadv.2009.05.026.

4. Kant, S., Kant, P., Raveh, E., et al. (2006). Evidence that differential gene expression between the halophyte, Thellungiella halophila, and Arabidopsis thaliana is responsible for higher levels of the compatible osmolyte proline and tight control of $\mathrm{Na}^{+}$uptake in T. halophila. Plant, Cell \& Environment, 29(7), 1220-1234. https ://doi.org/10.1111/j.1365-3040.2006.01502.x.

5. Katschnig, D., Bliek, T., Rozema, J., et al. (2015). Constitutive high-level SOS1 expression and absence of HKT1;1 expression in the salt-accumulating halophyte Salicornia dolichostachya. Plant Science, 234, 144-154. https://doi.org/10.1016/j.plant sci.2015.02.011.

6. Nawaz, I., Iqbal, M., Hakvoort, H. W., et al. (2014). Expression levels and promoter activities of candidate salt tolerance genes in halophytic and glycophytic Brassicaceae. Environmental and Experimental Botany, 99, 59-66. https://doi.org/10.1016/j.envex pbot.2013.10.006

7. Nawaz, I., Iqbal, M., Bliek, M., et al. (2017). Salt and heavy metal tolerance and expression levels of candidate tolerance genes among four extremophile Cochlearia species with contrasting habitat preferences. Science of the Total Environment, 584-585, 731-741. https://doi.org/10.1016/j.scitotenv.2017.01.111.

8. Møller, I. S., Gilliham, M., Jha, D., et al. (2009). Shoot $\mathrm{Na}^{+}$exclusion and increased salinity tolerance engineered by cell type-specific alteration of $\mathrm{Na}^{+}$transport in Arabidopsis. The Plant Cell, 21(7), 2163-2178. https://doi.org/10.1105/tpc.108.064568.

9. Plett, D. C., \& Møller, I. S. (2010). $\mathrm{Na}^{+}$transport in glycophytic plants: What we know and would like to know. Plant, Cell and Environment, 33(4), 612-626. https://doi.org/10.111 1/j.1365-3040.2009.02086.x.

10. Deinlein, U., Stephan, A. B., Horie, T., et al. (2014). Plant salttolerance mechanisms. Trend in Plant Science, 19(6), 371-379. https://doi.org/10.1016/j.tplants.2014.02.001.

11. Plett, D., Safwat, G., Gilliham, M., et al. (2010). Improved salinity tolerance of rice through cell type-specific expression of AtHKT1;1. PLoS ONE, 5(9), e12571. https://doi.org/10.1371/ journal.pone.0012571.

12. Inan, G., Zhang, Q., Li, P., et al. (2004). Salt Cress. A halophytes Arabidopsis relative model system and its applicability to molecular genetic analyses of growth and development of extremophiles. Plant Physiology, 135, 1718-1737. https://doi.org/10.1104/ pp.104.041723.

13. Ali, A., \& Yun, D.-J. (2016). Differential selection of sodium and potassium ions by TsHKT1;2. Plant Signaling \& Behavior, 11(8), e1206169. https://doi.org/10.1080/15592324.2016.1206169.

14. Ali, Z., Park, H. C., Ali, A., et al. (2012). TsHKT1;2, an HKT1 homolog from the extremophile Arabidopsis relative Eutrema salsugineum, shows $\mathrm{K}^{+}$specificity in the presence of $\mathrm{NaCl}$. Plant Physiology, 158, 1463-1474. https://doi.org/10.1104/ pp.111.193110.

15. Ali, A., Khan, I. U., Jan, M., et al. (2018). The high-affinity potassium transporter EpHKT1;2 from the extremophile Eutrema parvula mediates salt tolerance. Frontiers in Plant Science, 9, 1108. https://doi.org/10.3389/fpls.2018.01108.

16. Zhang, Y., Yin, H., Li, D., et al. (2008). Functional analysis of $B A D H$ gene promoter from Suaeda liaotungensis K. Plant Cell Reports, 27(3), 585-592. https://doi.org/10.1007/s0029 9-007-0459-8.

17. Sun, Q., Gao, F., Zhao, L., et al. (2010). Identification of a new $130 \mathrm{bp}$ cis-acting element in the TsVP1 promoter involved in the salt stress response from Thellungiella halophila. BMC Plant Biology, 10, 90. https://doi.org/10.1186/1471-2229-10-90.

18. Li, Q. L., Xie, J. H., Ma, X. Q., et al. (2016). Molecular cloning of Phosphoethanolamine $N$-methyltransferase (PEAMT) gene and its promoter from the halophyte Suaeda liaotungensis and their response to salt stress. Acta Physiologiae Plantarum, 38, 39. https ://doi.org/10.1007/s11738-016-2063-4.

19. Asins, J. M., Villalta, I., Aly, M., et al. (2013). Two closely linked tomato HKT coding genes are positional candidates for the major tomato QTL involved in $\mathrm{Na}^{+} / \mathrm{K}^{+}$homeostasis. Plant, Cell and Environment, 36(6), 1171-1191. https://doi.org/10.1111/ pce. 12051 .

20. Rus, A., Baxter, I., Muthukumar, B., et al. (2006). Natural variants of AtHKTl enhance $\mathrm{Na}^{+}$accumulation in two wild populations of Arabidopsis. PLoS Genetics, 2(12), 1964-1973. https://doi. org/10.1371/journal.pgen.0020210.

21. Baxter, I., Brazelton, J. N., Yu, D., et al. (2010). A coastal cline in sodium accumulation in Arabidopsis thaliana is driven by natural variation of the sodium transporter AtHKT1;1. PLoS Genetics, 6(11), e1001193. https://doi.org/10.1371/journal.pgen.1001193.

22. Schat, H., \& Ten Bookum, W. M. (1992). Genetic control of copper tolerance in Silene vulgaris. Heredity, 68, 219-229.

23. Jack, E., Hakvoort, H. W. J., Reumer, A., et al. (2007). Real-time PCR analysis of metallothionein-2b expression in metallicolous and non-metallicolous populations of Silene vulgaris (Moench) Garcke. Environmental and Experimental Botany, 59(1), 84-91. https://doi.org/10.1016/j.envexpbot.2005.10.005.

24. Rivera, R., Edwards, K. J., Barker, J. H., et al. (1999). Isolation and characterization of polymorphic microsatellites in Cocos nucifera L. Genome, 42(4), 668-675.

25. Mäser, P., Eckelman, B., Vaidyanathan, R., et al. (2002). Altered shoot/root $\mathrm{Na}^{+}$distribution and bifurcating salt sensitivity in Arabidopsis by genetic disruption of the $\mathrm{Na}^{+}$transporter AtHKT1 . FEBS Letters, 531(2), 157-161. https://doi.org/10.1016/S0014 -5793(02)03488-9.

26. Karimi, M., Inzé, D., \& Depicker, A. (2002). GATEWAYTM vectors for Agrobacterium-mediated plant transformation. Trends Plant Science, 7(5), 193-195. https://doi.org/10.1016/S1360 -1385(02)02251-3.

27. Clough, S. J., \& Bent, A. F. (1998). Floral dip: a simplified method for Agrobacterium-mediated transformation of Arabidopsis thaliana. The Plant Journal, 16(6), 735-743. https://doi.org/10.1046/ j.1365-313x.1998.00343.x.

28. Livak, K. J., \& Schmittgen, T. D. (2001). Analysis of relative gene expression data using Real-Time quantitative PCR and the $2^{-\Delta \Delta C T}$ method. Methods, 25(4), 402-408. https://doi.org/10.1006/ meth.2001.1262.

29. Higo, K., Ugawa, Y., Iwamoto, M., et al. (1988). PLACE: a database of plant cis-acting regulatory DNA elements. Nucleic Acids Research, 26(1), 358-359. https://doi.org/10.1093/nar/26.1.358.

30. Baek, D., Jiang, J., Chung, J. S., et al. (2011). Regulated AtHKT1 gene expression by a distal enhancer element and DNA methylation in the promoter plays an important role in salt tolerance. Plant and Cell Physiology, 52(1), 149-161. https://doi.org/10.1093/pcp/ pcq182.

31. Uozumi, N., Kim, E. J., Rubio, F., et al. (2000). The Arabidopsis HKT1 gene homolog mediates inward $\mathrm{Na}^{+}$currents in Xenopus laevis oocytes and $\mathrm{Na}^{+}$uptake in Saccharomyces cerevisiae. Plant Physiology, 122, 1249-1259. https://doi.org/10.1104/ pp.122.4.1249.

32. Berthomieu, P., Conejero, G., Nublat, A., et al. (2003). Functional analysis of AtHKT1 in Arabidopsis shows that $\mathrm{Na}^{+}$recirculation by the phloem is crucial for salt tolerance. The EMBO Journal, 22(9), 2004-2014. https://doi.org/10.1093/emboj/cdg207.

33. Sunarpi, Horie, T., Motoda, J., et al. (2005). Enhanced salt tolerance mediated by AtHKT1 transporter-induced $\mathrm{Na}^{+}$unloading from xylem vessels to xylem parenchyma cells. The Plant Journal, 44, 928-938. https://doi.org/10.1111/j.1365-313X.2005.02595.x. 
34. Vera-Estrella, R., Barkla, B. J., Garcıa-Ramırez, L., \& Pantoja, O. (2005). Salt stress in Thellungiella halophila activates $\mathrm{Na}^{+}$transport mechanisms required for salinity tolerance. Plant Physiology, 139, 1507-1517. https://doi.org/10.1104/pp.105.067850.

35. Volkov, V., \& Amtmann, A. (2006). Thellungiella halophila, a salt-tolerant relative of Arabidopsis thaliana, has specific root ionchannel features supporting $\mathrm{K}^{+} / \mathrm{Na}^{+}$homeostasis under salinity stress. Plant Journal, 48, 342-353. https://doi.org/10.1111/j.1365313X.2006.02876.x.

36. Tester, M., \& Davenport, R. (2003). $\mathrm{Na}^{+}$tolerance and $\mathrm{Na}^{+}$transport in higher plants. Annals of Botany, 91(5), 503-527. https:// doi.org/10.1093/aob/mcg058.

37. Yamaguchi-Shinozaki, K., \& Shinozaki, K. (1994). A novel cisacting element in an Arabidopsis gene is involved in responsiveness to drought, low-temperature, or high-salt stress. The Plant Cell, 6, 251-264. https://doi.org/10.1105/tpc.6.2.251.

38. Park, H. C., Kim, M. L., Kang, Y. H., et al. (2004). Pathogen- and $\mathrm{NaCl}$-induced expression of the SCaM-4 Promoter is mediated in part by a GT-1 Box that interacts with a GT-1-like transcription factor. Plant Physiology, 135, 2150-2161. https://doi.org/10.1104/ pp.104.041442.

39. Shirsat, A., Wilford, N., Croy, R., et al. (1989). Sequences responsible for the tissue specific promoter activity of a pea legumin gene in tobacco. Molelular and General Genetics, 215(2), 326331. https://doi.org/10.1007/BF00339737.

40. Behnam, B., Iuchi, S., Fujita, M., et al. (2013). Characterization of the promoter region of an Arabidopsis gene for 9-cis-epoxycarotenoid dioxygenase involved in dehydration-inducible transcription. DNA Research, 20(4), 315-324. https://doi.org/10.1093/dnares/ dst012.

41. Prabu, G. R., \& Parsad, D. T. (2011). Structure of DNA binding MYB transcription factor protein (SCMYBAS1-3) from sugarcane-Threading and AB initio modelling. Journal of Phytology, $3(3), 77-82$.

Publisher's Note Springer Nature remains neutral with regard to jurisdictional claims in published maps and institutional affiliations. 